\title{
Serotonin and the Small Cardioactive Peptides Differentially Modulate Two Motor Neurons That Innervate the Same Muscle Fibers in Aplysia
}

\author{
Lyle E. Fox and Philip E. Lloyd \\ Committee on Neurobiology and Department of Pharmacological and Physiological Sciences, University of Chicago, \\ Chicago, Illinois 60637
}

The anterior portion of intrinsic buccal muscle 3 (I3a) is innervated by two motor neurons, B3 and B38, which appear to use glutamate as their fast excitatory transmitter. B3 and B38 express the neuropeptides FMRFamide and the small cardioactive peptides (SCPs), respectively. We have shown previously that stimulation of B38 causes release of the SCPs from terminals in the muscle. The 13 a muscle also receives input from neurons that use $5 \mathrm{HT}$ as a modulatory transmitter. The SCPs and $5 \mathrm{HT}$ potently facilitated B38-evoked excitatory junction potentials (EJPs) but had only a small effect on B3-evoked EJPs; however, both the SCPs and $5 \mathrm{HT}$ strongly potentiated contractions evoked by both B3 and B38, indicating that the two substances must also act on excitation-contraction coupling. The selective facilitation of B38-evoked EJPs, however, did manifest itself in other parameters. Decreases in the firing frequencies and burst durations that were threshold to evoke contractions and decreases in the latency between the onset of a burst and the onset of the evoked contraction were all much larger for B38 than for B3. Indeed, B38 bursts recorded during feeding-like behavior would be subthreshold for evoking contractions in the absence of this modulation. All of the effects of the SCPs reversed during washout, whereas those of $5 \mathrm{HT}$ were persistent, lasting many hours after washout. Thus, the SCPs and $5 \mathrm{HT}$ dramatically change the behavioral output of these motor neurons, increasing the amplitude of contractions evoked by both B3 and B38, and shifting the temporal relationship between bursts in B38 and its evoked contractions.

Key words: neuropeptide; facilitation; contraction; potentiation; synapse; latency
Modulation of synaptic transmission has proven to be a powerful means by which the nervous system controls synaptic strength. In invertebrates, modulation of synapses between neurons and muscle fibers seems to be particularly widespread (Calabrese, 1989). In Aplysia, neuromuscular synapses between motor neurons in the buccal ganglia and their target muscles have been used extensively to study modulation. These motor neurons produce the cyclic motor output that drives ingestive and egestive movements of muscles of the buccal mass (Gardner, 1971; Kupfermann, 1974). These motor neurons have discrete targets and use either acetylcholine or glutamate as their fast transmitters and many neuropeptides as intrinsic modulatory cotransmitters (Cropper et al., 1987a,b, 1988, 1994; Lloyd et al., 1987; Church and Lloyd, 1991; Fox and Lloyd, 1993; Lloyd and Church, 1994). These peptides are transported by fast axonal transport from neurons in the buccal ganglia to terminals in muscle (Lloyd, 1988), where they are released and are capable of modulating evoked contractions (Whim and Lloyd, 1989, 1990; Cropper et al., 1990). In addition, an identified purely modulatory neuron releases 5HT, which acts as an extrinsic modulator of these neuromuscular synapses (Weiss et al., 1978; Lotshaw and Lloyd, 1990).

Because the expression of intrinsic peptide cotransmitters was determined in many identified buccal neurons, it was possible to

\footnotetext{
Received Feb. 28, 1997; revised May 27, 1997; accepted June 2, 1997.

This work was supported by National Research Service Award 1-F31-MH10656 to L.E.F. and National Science Foundation Grant BNS-9418815 to P.E.L. We thank Christopher Keating for critical reading of this manuscript.

Correspondence should be addressed to Philip E. Lloyd, Committee on Neurobiology, University of Chicago, 947 E. 58th Street, Chicago, IL 60637.

Copyright (C) 1997 Society for Neuroscience $0270-6474 / 97 / 176064-\bullet \$ 05.00 / 0$
}

choose a preparation amenable for studying the role of these transmitters (Church and Lloyd, 1991). The preparation we chose consists of the anterior intrinsic muscle 3 (I3a), which is innervated by excitatory motor neurons B3 and B38, which likely use glutamate as their fast transmitter; inhibitory motor neuron B47, which uses acetylcholine as its fast transmitter; and the modulatory serotonergic neuron (termed the metacerebral cell or MCC) (Weiss et al., 1978; Lotshaw and Lloyd, 1990). All three motor neurons express peptide cotransmitters: B3 expresses FMRFamide, B38 the SCPs, and B47 the myomodulins (Church et al., 1993). Thus, these neuromuscular synapses are modulated by neuropeptides, which are intrinsic to the motor innervation, and by $5 \mathrm{HT}$, which is extrinsic to the motor innervation (Weiss et al., 1978; Lloyd et al., 1984; Cropper et al., 1987a, 1990; Whim and Lloyd, 1990). We have previously used B3-evoked excitatory junction potentials (EJPs) and muscle contractions to assay heterosynaptic modulation caused by neuropeptide release from B38 and B47 (Church et al., 1993) and have shown that stimulation of the MCC, or application of the SCPs and 5HT, facilitated B38evoked EJPs and potentiated contractions (Lotshaw and Lloyd, 1990). Thus, two modulatory substances seem to have very similar effects on this preparation. This raises the possibility that intrinsic and extrinsic modulators may have identical actions and be functionally redundant. We wished to test this hypothesis thoroughly at the I3a neuromuscular system by quantitatively comparing the effects of the SCPs and 5HT on the EJPs and contractions evoked by B3 and B38. Even relatively subtle differences in the actions of two modulatory substances may greatly increase the flexibility with which a synapse can be regulated (Brezina et al., 1996). 
A

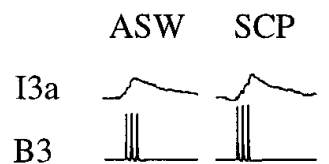

B3

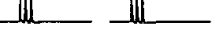

I3a


Figure 1. Effects of $1 \mu \mathrm{M} \mathrm{SCP}$ or $1 \mu \mathrm{M} 5 \mathrm{HT}$ on EJPs recorded intracellularly in I3a muscle fibers. A, Selective effects of SCPs and 5HT on EJPs evoked by B3 and B38. Note that B38-evoked EJPs were facilitated markedly by both the SCPs and 5HT, whereas these substances had little effect on the amplitude of B3-evoked EJPs. $B$, Time courses of the effects of the SCPs and 5HT on B38-evoked EJPs. Records for 5HT or the SCPs are each from a single muscle fiber. Application of the SCPs caused a 4 $\mathrm{mV}$ hyperpolarization from a rest potential of $-72 \mathrm{mV}$; $5 \mathrm{HT}$ caused an 8 $\mathrm{mV}$ hyperpolarization from a rest of $-78 \mathrm{mV}$. Note that the effects of the SCPs reversed during washout, whereas those of $5 \mathrm{HT}$ persisted for at least $3 \mathrm{hr}$.

\section{MATERIALS AND METHODS}

Animals. Aplysia californica (50-150 gm) were obtained from Marinus Inc. (Long Beach, CA), maintained in circulating artificial seawater (ASW) at $16^{\circ} \mathrm{C}$, and fed dried seaweed every $3 \mathrm{~d}$.

Motor neuron stimulation experiments. Animals were immobilized with an injection of isotonic $\mathrm{MgCl}_{2}$ and dissected in either low $\mathrm{Ca}^{2+}(0.5 \mathrm{~mm}$; $0.05 \times$ normal $)$, high $\mathrm{Mg}^{2+}(110 \mathrm{mM} ; 2 \times$ normal $)$ ASW (termed low Ca ASW), or high $\mathrm{Ca}^{2+}(33 \mathrm{~mm} ; 3 \times$ normal $)$, high $\mathrm{Mg}^{2+}(165 \mathrm{~mm} ; 3 \times$ normal) ASW (termed high $\mathrm{Ca}, \mathrm{Mg} \mathrm{ASW}$ ). The buccal mass/buccal ganglia complex was removed and bisected along the midline, and all nerves were severed except ipsilateral buccal nerve 2 (nerve designations, Gardner, 1971; muscle nomenclature, Howells, 1942; also see Lloyd, 1988). The hemiganglion was desheathed and selectively superfused with either low Ca ASW to suppress synaptic transmission in the ganglion or high $\mathrm{Ca}, \mathrm{Mg}$ ASW to raise the firing thresholds of neurons in the ganglion. The remainder of the bath containing the I3a muscle was separated by a barrier (except when a perfusion electrode was used; see below) through which the intact nerve 2 ran and was superfused with ASW. The SCPs or 5HT in ASW were applied selectively to the bath containing the muscle so that the ganglion was not exposed to these substances.

In typical experiments, both the SCPs and 5HT were applied to the same muscle. Because the effects of the SCPs were reversible and the effects of 5HT were persistent, the SCPs were applied to the muscle first and washed out before 5HT was applied. Additional experiments were also performed in which only $5 \mathrm{HT}$ was applied to the muscle. $\mathrm{SCP}_{\mathrm{A}}$ was
A
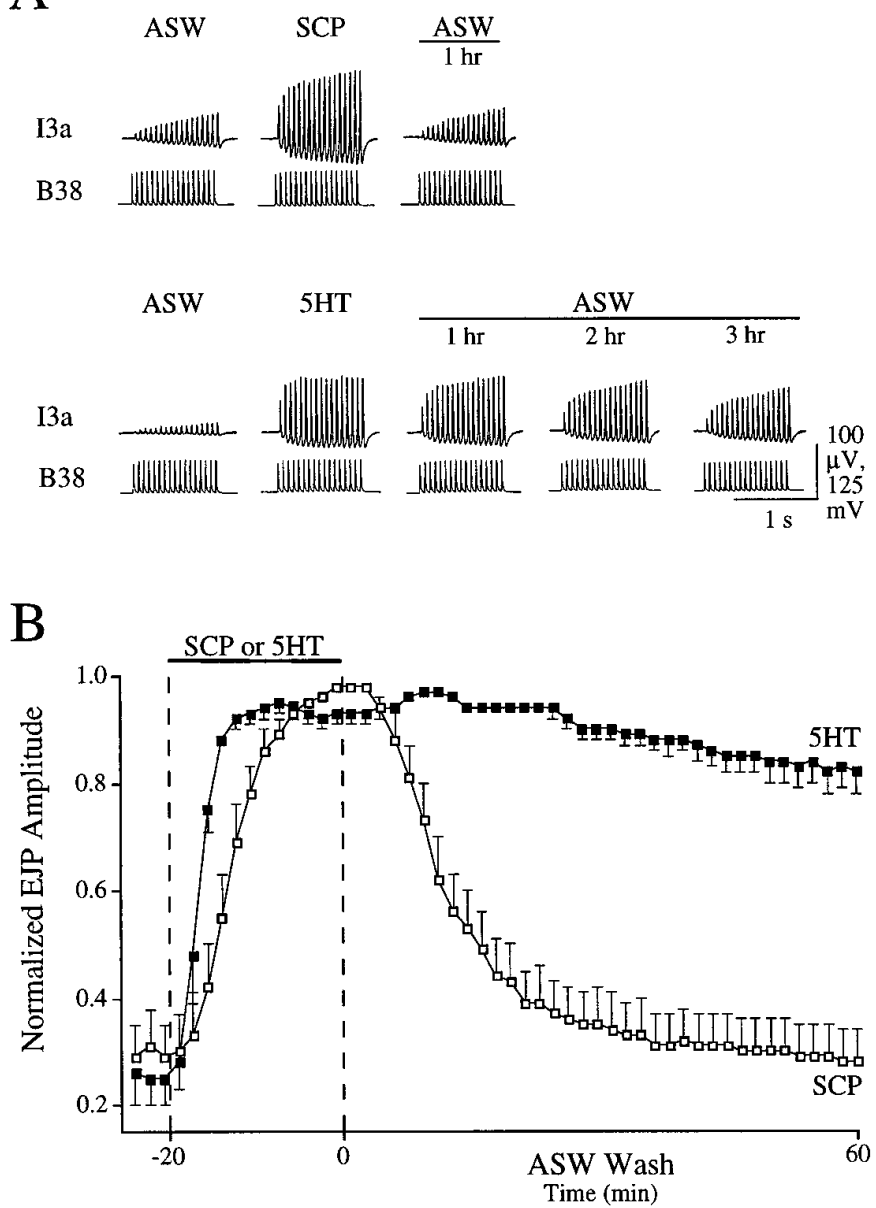

Figure 2. Effects of $1 \mu \mathrm{M} \mathrm{SCP}$ or $1 \mu \mathrm{M} 5 \mathrm{HT}$ on EJPs recorded extracellularly in I3a muscle using a perfusion electrode. $A$, Effects of SCPs and 5HT on EJPs evoked by stimulating B38. Note that the effects of the SCPs reversed during washout, whereas those of $5 \mathrm{HT}$ persisted for at least $3 \mathrm{hr}$. Recordings are from different experiments. $B$, Cumulative results for the SCPs and 5HT on B38-evoked EJPs $(n=11)$. To pool results from different neuromuscular preparations, EJPs were normalized to the maximum EJP amplitude (set at 1.0). 5HT or the SCPs were applied from -20 to $0 \mathrm{~min}$. Note that the effects of the SCPs reverse during washout, whereas those of 5HT persisted for at least $1 \mathrm{hr}$ of washout. The small upward inflection of EJP amplitude observed at the end of 5HT perfusion suggests that 5HT may also have a small inhibitory effect that reverses during washout.

routinely used in this study, although occasional tests using $\mathrm{SCP}_{\mathrm{B}}$ indicated that the two peptides had identical activities (Lloyd, 1986). Neurons were impaled with two microelectrodes (2-4 M 2 ; filled with $3 \mathrm{M}$ potassium acetate, $0.1 \%$ fast green), one to inject current and one to monitor membrane potential. B3 and B38 were identified by their position, size, nature of synaptic input, and muscle innervation patterns (Church et al., 1993). Whenever possible, the data presented in this paper were generated from experiments in which both B3 and B38 were alternately stimulated in the same preparation. This experimental design was used to reduce the variability between preparations. Additional experiments using only B3 or B38 verified that the results were not caused by interactions between the two neurons. For example, using data from intracellular recordings, the SCPs $(1 \mu \mathrm{M})$ increased B38-evoked EJPs $417 \pm 80 \%(n=7)$ over control when all experiments were pooled, and $433 \pm 145 \%(n=3)$ when only the experiments in which both motor neurons were alternately stimulated were pooled.

Measurement of I3a EJPs. Individual spikes in motor neurons were driven by brief $(10-20 \mathrm{msec})$ depolarizing current pulses. EJPs were recorded with an intracellular electrode $(\sim 20 \mathrm{M} \Omega$; filled as described above) or via a perfusion electrode (see below). For intracellular muscle 
A
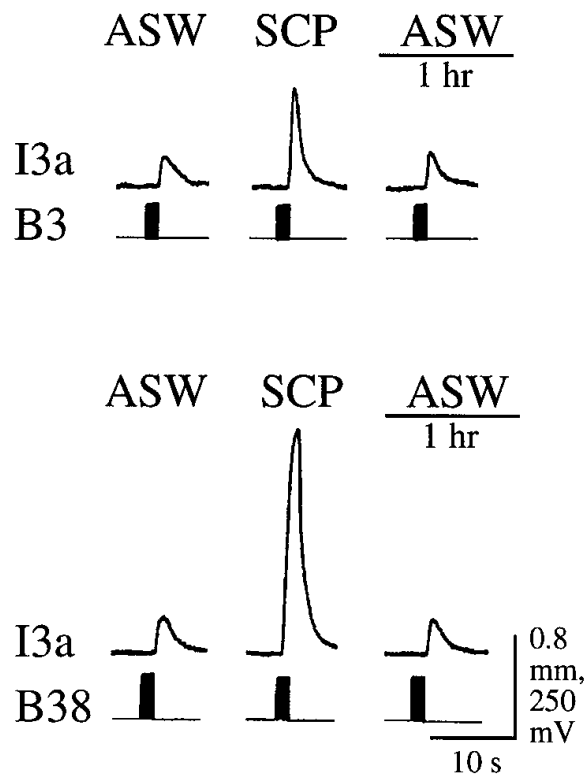

B
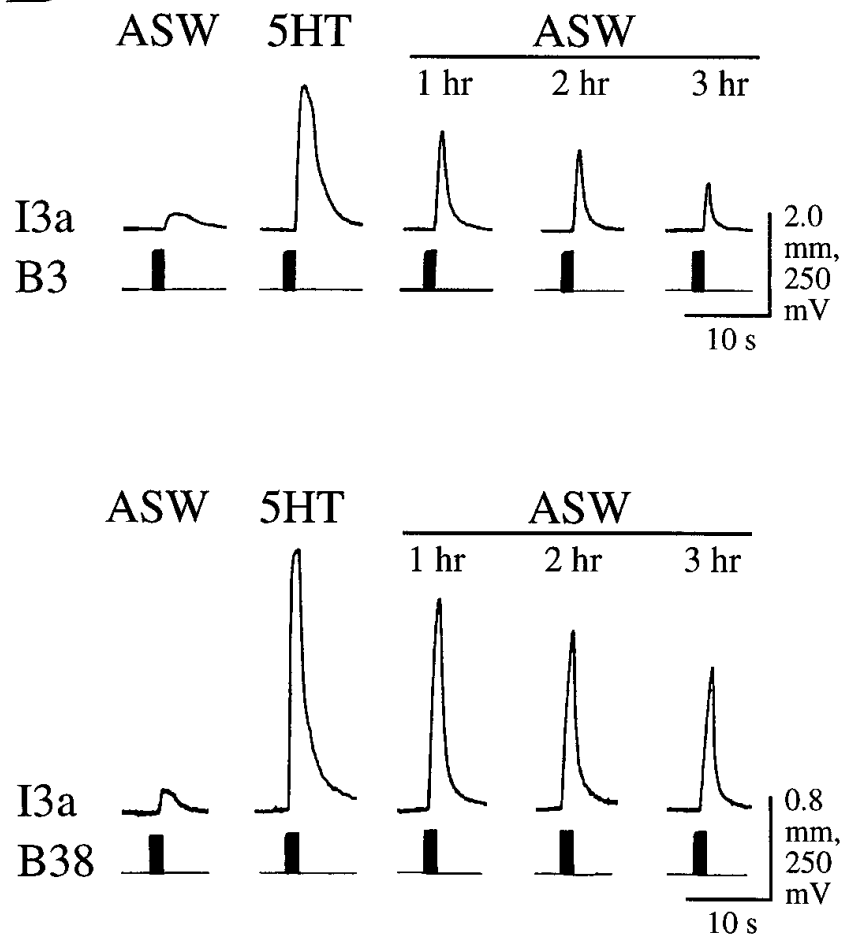

Figure 3. Effects of the SCPs and 5HT on I3a muscle contractions. A, Effects of $1 \mu \mathrm{M} \mathrm{SCP}$ on contractions evoked by stimulating $\mathrm{B} 3$ or B38. Bursts were alternately fired in B3 or B38 $(16 \mathrm{~Hz}$ for $1.6 \mathrm{sec}$ at interburst interval of $100 \mathrm{sec}$ ). The first contraction is in ASW, the second is after 20 min superfusion with the SCPs, and the third is after $1 \mathrm{hr}$ wash in ASW. Note that the effects of the SCPs reverse during washout. $B$, Effects of 1 $\mu \mathrm{M} 5 \mathrm{HT}$ on contractions evoked by alternately stimulating B3 or B38. Bursts were alternately fired in B3 (16 Hz for $1.3 \mathrm{sec}$ at interburst interval of $100 \mathrm{sec})$ or $\mathrm{B} 38(16 \mathrm{~Hz}$ for $1.6 \mathrm{sec}$ at interburst interval of $100 \mathrm{sec})$. Note that the effects of $5 \mathrm{HT}$ persisted for at least $3 \mathrm{hr}$. Recordings are from different experiments.
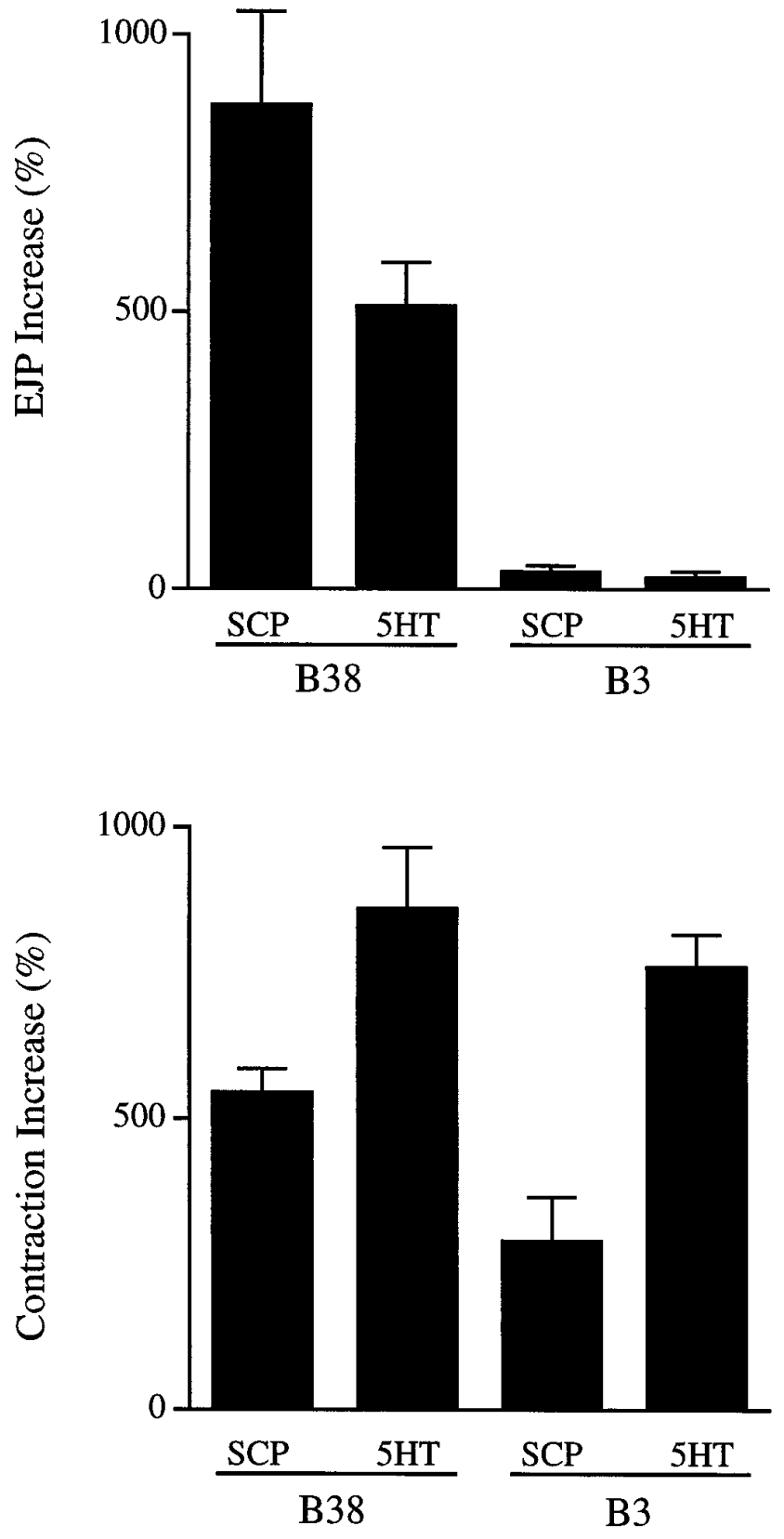

Figure 4. Summary of the effects of the SCPs and 5HT on extracellularly recorded EJPs and contractions. Top, The effect of $1 \mu \mathrm{M}$ SCPs or $1 \mu \mathrm{M}$ $5 \mathrm{HT}$ on EJPs evoked by the stimulation of B3 or B38. The mean amplitude of the third EJP in the bursts was used because the first EJP was often too small to measure reliably (e.g., Fig. 2). The B38-evoked EJPs were dramatically facilitated by both the SCPs $(n=12 ; p \leq 0.01 ; t$ test) and $5 \mathrm{HT}(n=11 ; p \leq 0.01)$, whereas the B3-evoked EJPs were only slightly facilitated by the SCPs $(n=12 ; p \leq 0.01)$ or $5 \mathrm{HT}(n=10$; not significant). The SCPs were more effective at increasing EJPs than 5HT (B3, $p \leq 0.05$; paired $t$ test; B38, $p \leq 0.01$ ). Bottom, The effect of $1 \mu \mathrm{M}$ SCPs or $1 \mu \mathrm{M} 5 \mathrm{HT}$ on contractions evoked by the stimulation of $\mathrm{B} 3$ or B38. Both the SCPs and 5HT increased the amplitude of contractions evoked by both motor neurons $(n=4 ; p \leq 0.01)$. Note that 5 HT was more effective than the SCPs at potentiating contractions $(p \leq 0.01)$ and that the SCPs were more effective at potentiating contractions evoked by B38 than those evoked by B3 $(p \leq 0.05)$. The values for the effects of the SCPs on the amplitude of B3-evoked EJPs and contractions are very similar to those reported previously (Church et al., 1993). 


\section{B3}

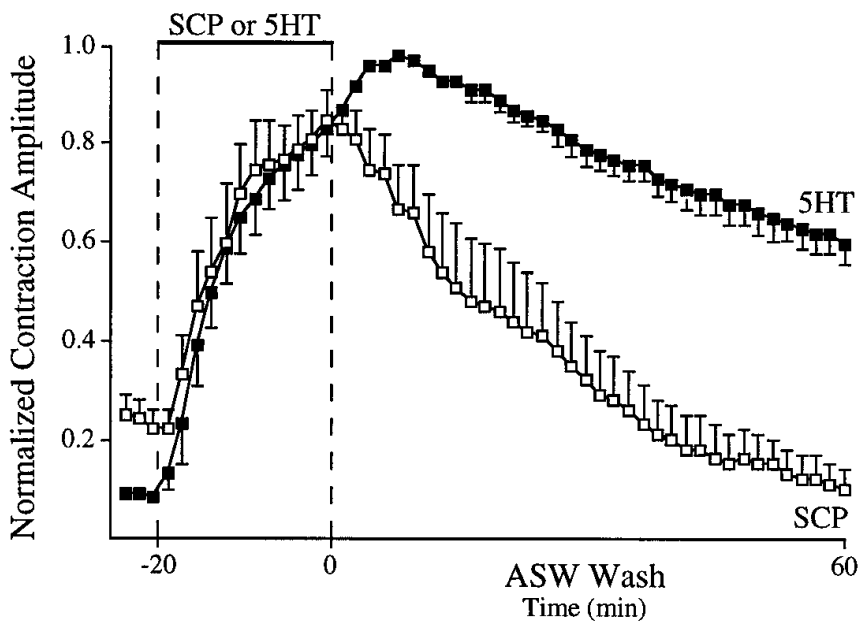

\section{B38}

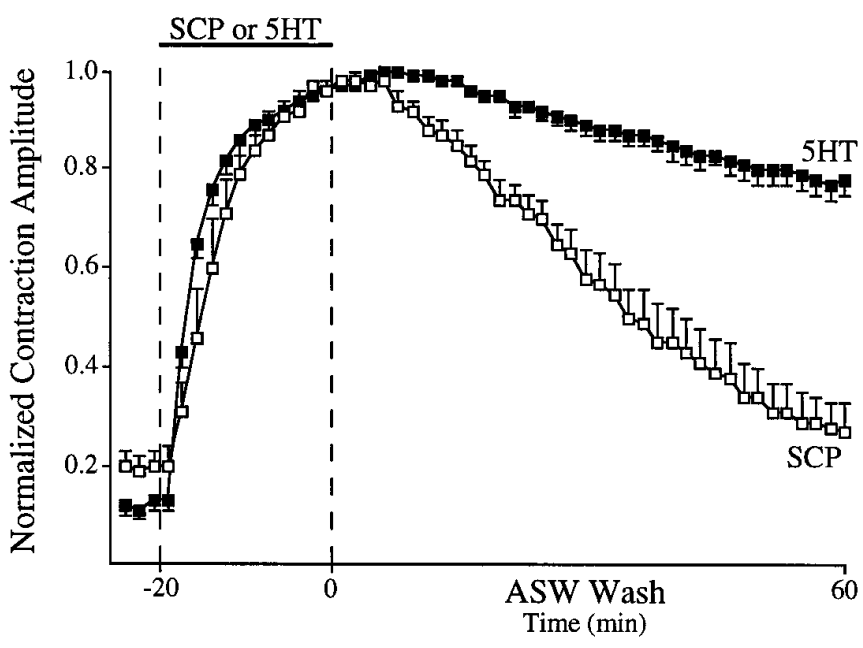

Figure 5. Cumulative results for the effects of the SCPs and 5HT on motor neuron-evoked contractions. Top, Time course of the effects of SCPs and 5HT on B38-evoked contractions $(n=7)$. Bottom, Time course of the effects of SCPs and 5HT on B3-evoked contractions $(n=8)$. To pool results from different preparations, contraction amplitudes were normalized to the maximum contraction amplitude (set at 1.0). $\mathrm{SCP}_{\mathrm{A}}(1$ $\mu \mathrm{M})$ or $5 \mathrm{HT}(1 \mu \mathrm{M})$ were applied from -20 to $0 \mathrm{~min}$. Note that the effects of the SCPs reversed during washout, whereas those of $5 \mathrm{HT}$ persisted for at least $1 \mathrm{hr}$ of washout.

fiber recordings, short bursts of three to seven action potentials at 12.5 $\mathrm{Hz}$ were used to ensure that a burst did not evoke contractions. The perfusion electrode consisted of a small chamber $(100 \mu \mathrm{l})$ and aperture $(\sim 1.5 \mathrm{~mm})$, which was positioned to press firmly down on a portion of the muscle (Church et al., 1993, their Fig. 1). The inside of the chamber was superfused rapidly with ASW $(1.5 \mathrm{ml} / \mathrm{min})$. The remainder of the muscle outside of the recording chamber was superfused with low Ca ASW to suppress synaptic transmission and muscle contractions. This procedure confined the contractions to the small area of the muscle covered by the recording chamber and thus markedly reduced movement artifacts in the recordings. The earliest evoked muscle contractions occur after the sixth EJP so that the early EJPs in a burst are recorded in the absence of any movement. Stimulation at $16 \mathrm{~Hz}$ was used routinely in these experiments. EJPs were recorded by extracellular electrodes placed inside and just outside the wall of the perfusion apparatus. Signals were amplified using a Grass P15D AC amplifier. The SCPs or 5HT were applied in ASW to the inner chamber of the perfusion electrode so that the ganglia were not exposed to these substances. Typical application periods were $20 \mathrm{~min}$ to
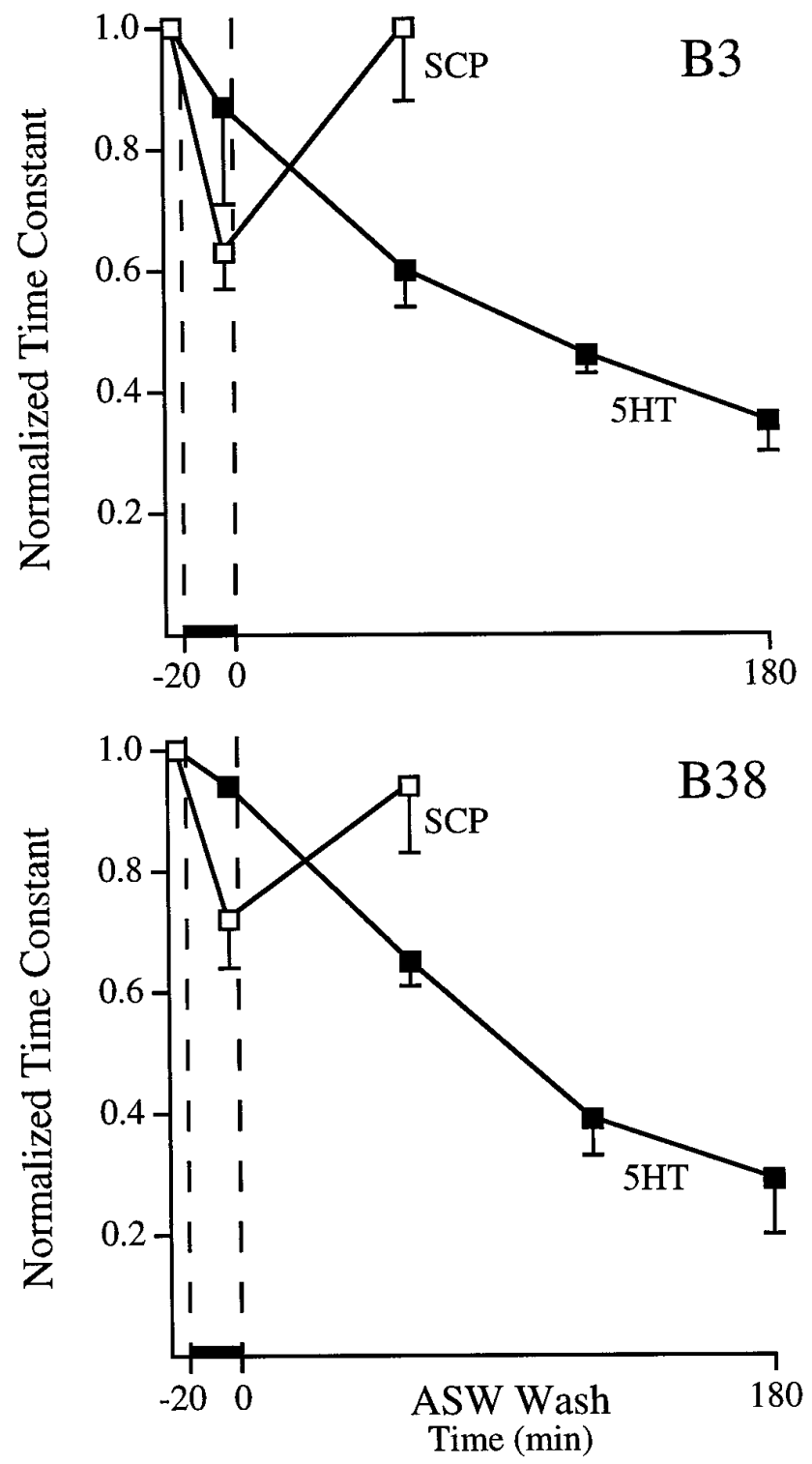

Figure 6. Cumulative results for the effects of SCPs and 5HT on relaxation time constants of motor neuron-evoked contractions. Top, Time course of the effects of SCPs and 5HT on the relaxation time constants of B38-evoked contractions $(n=3)$. Bottom, Time course of the effects of SCPs and 5HT on the relaxation time constants of B3-evoked contractions (SCPs, $n=4$; 5HT, $n=3$ ) To pool results from different neuromuscular preparations, relaxation time constants were normalized to control (set at 1.0; decreasing time constants indicate more rapid relaxation rates). $\mathrm{SCP}_{\mathrm{A}}(1 \mu \mathrm{M})$ or $5 \mathrm{HT}(1 \mu \mathrm{M})$ were applied from -20 to $0 \mathrm{~min}$ (indicated by black bars). Note that the effects of the SCPs reverse during washout, whereas those of 5HT continued to increase throughout the washout. It is difficult to interpret the relaxation rate results in light of the large changes in contraction amplitude, but it is clear that the effects of $5 \mathrm{HT}$ on amplitude and relaxation rate were persistent.

ensure adequate penetration into the muscle. Experiments were performed at room temperature $\left(\sim 22^{\circ} \mathrm{C}\right)$. In most experiments, long interburst intervals $(100 \mathrm{sec})$ were used to minimize release of endogenous SCPs from B38 or FMRFamide from B3 and to minimize post-tetanic potentiation (Lotshaw and Lloyd, 1990; Whim and Lloyd, 1990; Church et al., 1993).

Measurement of motor neuron-evoked I3 a muscle contractions. Neurons were impaled and stimulated as described above. Reproducible submaximal contractions were evoked by stimulating B3 or B38 with an interburst interval of $100 \mathrm{sec}$. The frequency of action potentials within a burst and 
A

\section{ASW SCP}

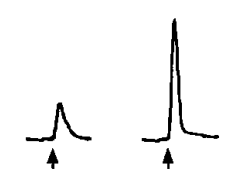

B
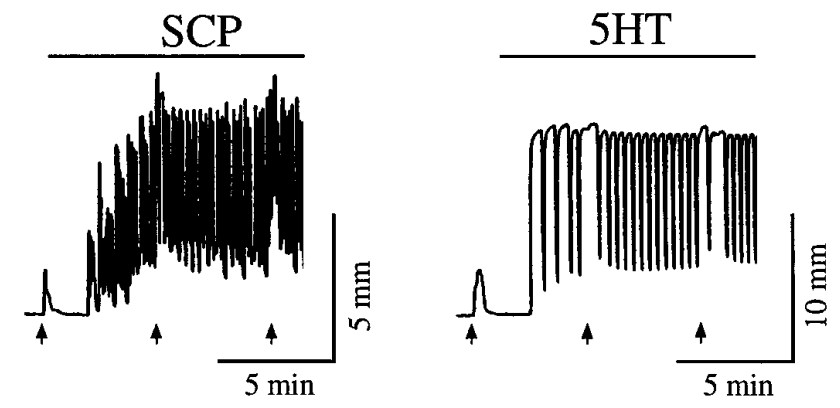

Figure 7. Effects of the SCPs and 5HT on I3a muscle contractions evoked by bolus applications of glutamate. $A$, Superfusion with $0.1 \mu \mathrm{M}$ $5 \mathrm{HT}$ or $0.1 \mu \mathrm{M} \mathrm{SCP} \mathrm{A}_{\mathrm{A}}$ increased the contractions evoked by boluses of 100 nmol glutamate (upward arrows). The first contraction is in ASW, the second is after 15 min superfusion with the SCPs or 5HT. B, Superfusion of higher concentrations $(1 \mu \mathrm{M})$ of the SCPs or 5HT (horizontal bar) caused I3a muscles to contract rhythmically.

burst duration varied with the experiment. Contraction amplitudes were monitored with an isotonic transducer (Harvard Apparatus). The SCPS or 5HT were applied selectively to the muscle via the superfusion in ASW as described above.

Measurement of glutamate-evoked I3a muscle contractions. I3a muscle fibers are arranged in bundles, and a few bundles were isolated in these experiments to enhance penetration of glutamate. Reproducible submaximal contractions were evoked by bolus applications $(5-10 \mu \mathrm{l})$ of L-glutamate (1-30 mM) injected into a $300 \mu \mathrm{l}$ bath at a flow rate of 1.5 $\mathrm{ml} / \mathrm{min}$ at $5 \mathrm{~min}$ intervals. Contractions were measured with an isotonic transducer, and the SCPs and 5HT were applied as described above.

\section{RESULTS}

\section{Effects of the SCPs and 5HT on EJPs evoked by B3 and B38}

I3a muscle fibers are electrically coupled to one another and are all functionally innervated by both B3 and B38 (Church et al., 1993). The fibers are nonspiking, and bursts of action potentials in the motor neurons produce compound EJPs that cause contractions after a threshold voltage is reached (Cohen et al., 1978; Lotshaw and Lloyd, 1990). Although the muscle fibers are small (5-10 $\mu \mathrm{m}$ in diameter), it is possible to record intracellularly from them while stimulating motor neurons as long as stimulation parameters (firing frequency and burst duration) are kept below the threshold for contractions. In most experiments, the SCPs and $5 \mathrm{HT}$ were applied at $1 \mu \mathrm{M}$, a concentration at which the two substances caused similar increases in cAMP levels of I3a muscles ( $\sim 20$-fold increase) (Lotshaw and Lloyd, 1990), and thus this seemed the most appropriate concentration at which to compare their effects. In experiments in which both B3 and B38 were alternately stimulated, application of $1 \mu \mathrm{M}$ SCPs selectively to the muscle produced very little change in the amplitude of EJPs evoked by B3 (an increase of $9 \pm 8 \%$ over control; SEM; $n=3$ ), whereas EJPs evoked by B38 in the same fibers were markedly facilitated (an increase of $433 \pm 145 \% ; n=3$ ) (Fig. 1). These values were obtained using the third EJPs in the bursts (which often consisted of only three spikes), because the first EJPs in ASW were often too small to measure accurately. Decay of compound EJPs appears to be passive and can be well fitted to a single exponential. The SCPs reduced decay time constants for EJPs evoked by both B3 (a decrease of $47 \pm 7 \%$ ) and B38 (a decrease of $51 \pm 13 \%$ ), suggesting a decrease in the input resistance of the muscle fibers as was reported previously (Lotshaw and Lloyd, 1990). All of the effects of the SCPs reversed during washout. Application of $1 \mu \mathrm{M} 5 \mathrm{HT}$ produced effects similar to those of the SCPs (Fig. 1). 5HT caused little change in B3-evoked EJPs (an increase of $22 \pm 21 \% ; n=5$ ) and a large facilitation of B38-evoked EJPs (an increase of $531 \pm 172 \% ; n=5$ ). 5HT decreased EJP decay time constants for both B3 (a decrease of $43 \pm 9 \%$ ) and B38 (a decrease of $42 \pm 8 \%$ ); however, the facilitation of B38-evoked EJPs produced by $5 \mathrm{HT}$ did not reverse on washout and lasted as long as impalements were held (up to 3 $\mathrm{hr}$ ). The effects of 5HT on B3-evoked EJPs were too small to determine whether they were also persistent.

Another method of recording EJPs was also used. This procedure permits stable long-term recordings and rapid solution turnover, and it records from a population of fibers simultaneously, thereby reducing sampling bias. A perfusion electrode was used to record extracellular EJPs in a small portion of the I3a muscle that is perfused with ASW while the remainder of the muscle is superfused with low Ca ASW to suppress synaptic transmission and muscle contractions (Church et al., 1993). Results from this technique were similar to those obtained with intracellular electrodes (compare Figs. $1 B$ and $2 A$ ). Pooling the third EJPs from experiments in which $\mathrm{B} 3$ and $\mathrm{B} 38$ were alternately stimulated, the SCPs and 5HT dramatically facilitated B38-evoked EJPs, whereas they had little effect on B3-evoked EJPs (summarized in Fig. 4). The SCPs were more effective than 5HT in facilitating B38-evoked EJPs. Again, the effects of the SCPs on B38-evoked EJPs reversed during washout, whereas those of $5 \mathrm{HT}$ were persistent (Fig. 2B).

\section{Effects of the SCPs and 5HT on contractions evoked by $\mathrm{B} 3$ and $\mathrm{B} 38$}

Because the SCPs and 5HT selectively facilitated B38-evoked EJPs, one would expect that they might selectively increase the amplitude of contractions evoked by B38 compared with those evoked by B3; however, the SCPs and 5HT dramatically potentiated contractions evoked by both motor neurons (Fig. 3). Therefore, the potentiation of contractions differed significantly from the facilitation of EJPs (Fig. 4). The SCPs and 5HT facilitated B38-evoked EJPs much more than B3-evoked EJPs, whereas contractions evoked by B3 and B38 were potentiated to a similar degree. Also, the SCPs were more effective than $5 \mathrm{HT}$ at facilitating EJPs, but 5HT was more effective at potentiating contractions. An explanation for these observations is that the SCPs and 5HT potentiate contractions at multiple sites. One site is the selective facilitation of B38-evoked EJPs by the SCPs and 5HT, whereas the other sites are in processes interposed between the EJPs and muscle contractions, i.e., in the processes that mediate excitation-contraction coupling. At these sites, 5HT must be more effective than the SCPs. The SCPs and 5HT also caused an increase in the relaxation rate of the contractions in the I3a muscle (Figs. 3, 5). These relaxation time constants could be well fitted to a single exponential and were measured in regions in 
A

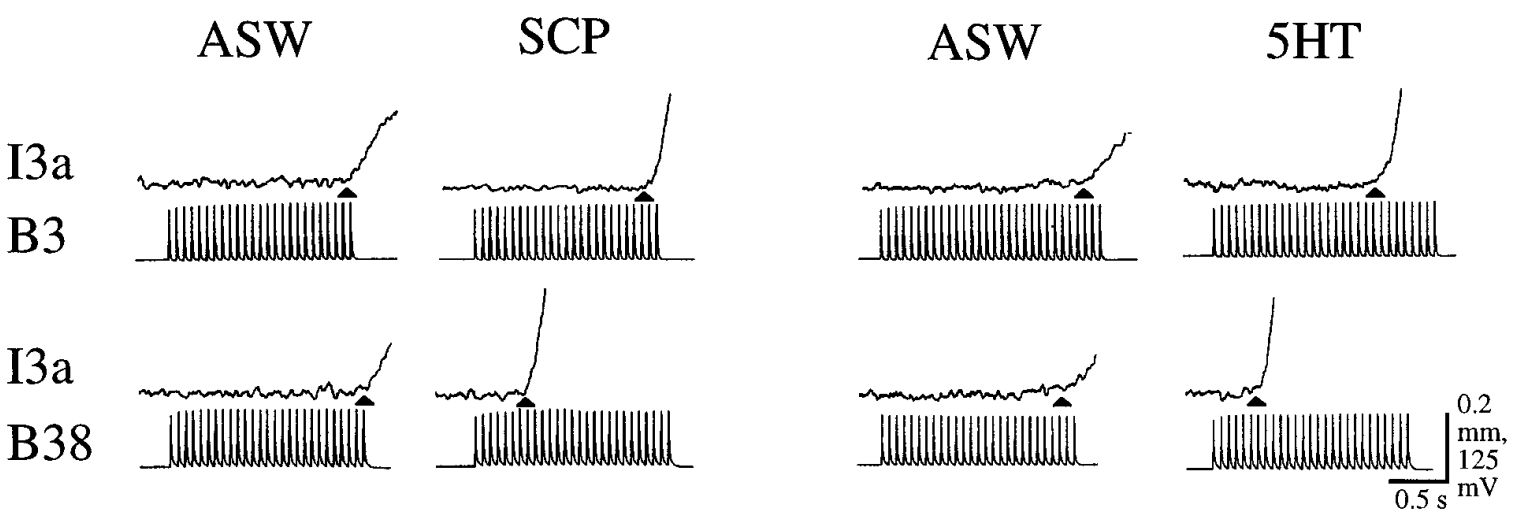

B

I3a

B38
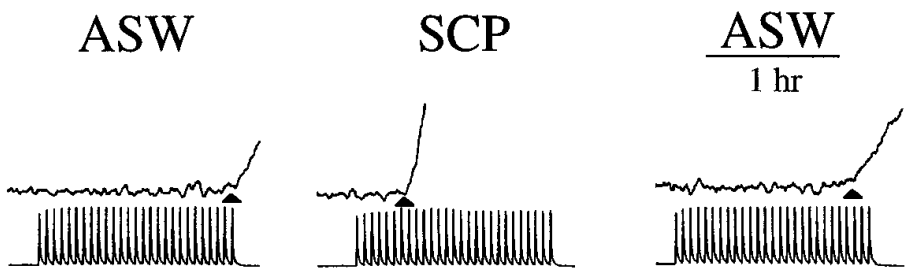

ASW

$5 \mathrm{HT}$

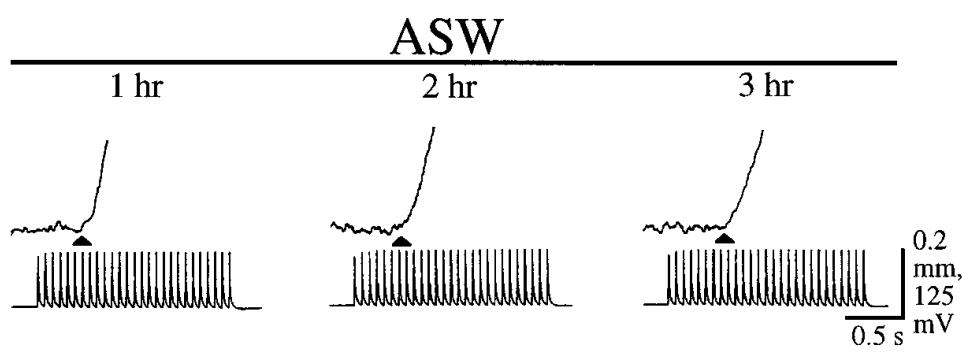

Figure 8. Effects of the SCPs and 5HT on the latency between the onset of a motor neuron burst and the onset of the resulting I3a contraction. $A$, Muscle contractions were evoked by stimulation of B3 or B38. Bursts of spikes were fired alternatively in B3 or B38 (16 Hz for 1.6 sec at interburst interval of $100 \mathrm{sec}$ ) for the application of the SCPs. B3 bursts were increased to $1.7 \mathrm{sec}$ before application of 5HT so that the contractions evoked by B3 and B38 would be of similar amplitude. The timing of the onset of muscle contractions is emphasized by recording at a fast time base and high gain. As a result, most of the contractions are off scale. Superfusion with either $1 \mu \mathrm{M} \mathrm{SCP}$ or $1 \mu \mathrm{M} 5 \mathrm{HT}$ increased the amplitude of muscle contractions evoked by both B3 and B38 (visible as an increased rate of rise in the contraction traces; also see Fig. 3); however, the reduction in latency between the onset of a motor neuron burst and the onset of the resulting contraction was reduced much more dramatically for contractions evoked by B38 than it was for contractions evoked by B3. B, The effects of 5HT are persistent, whereas those of the SCPs reverse during washout. The first contraction is in ASW, and the second is after $20 \mathrm{~min}$ superfusion with either $1 \mu \mathrm{M} 5 \mathrm{HT}$ or $1 \mu \mathrm{M} \mathrm{SCP}$. Subsequent contractions are after washout in ASW for the indicated periods. All recordings were from a single experiment. Black arrowheads indicate the onset of the contraction.

which the amplitude of control and potentiated contractions overlapped. Because contractions evoked by both B3 and B38 were strongly potentiated, it was possible to measure the time courses for both neurons. The potentiation of muscle contractions evoked by both motor neurons reversed during washout for the SCPs and were persistent for 5HT (Fig. 5). Indeed, the increase in relaxation rate caused by $5 \mathrm{HT}$ appeared to continue to increase progressively from the onset of 5HT perfusion to the end of washout over at least $3 \mathrm{hr}$ (Fig. 6). It is difficult, however, to interpret the relaxation rate results in light of the large changes in contraction amplitude, but it is clear that the effects of 5HT on amplitude and relaxation rate were certainly persistent.

Some results reported here appear to differ from previous work on this preparation. Chief among these is that the effects of $5 \mathrm{HT}$ $(0.5 \mu \mathrm{M})$ on B38-evoked EJPs and contractions were previously reported to be reversible (Lotshaw and Lloyd, 1990) and in the present study were found to be persistent. We believe that the major reason for the different results was that longer applications of 5HT (routinely $20 \mathrm{~min}$ ) were used in the present study and accessibility to the muscle fibers was improved. Like Lotshaw and Lloyd (1990), we also observed that the effects of 5HT were reversible with brief application $(<5 \mathrm{~min})$ of $5 \mathrm{HT}(1 \mu \mathrm{M})$.

Additional experiments were performed to determine whether some aspects of the potentiation of contractions caused by the SCPs and 5HT were also mediated postsynaptically. In these experiments, motor neuron-evoked transmitter release was sub- 
stituted with exogenous application of glutamate, and only small pieces of the I3a muscle were used to improve penetration. At 1 $\mu \mathrm{M}$, the SCPs and 5HT elicited spontaneous rhythmic contractions in the absence of glutamate applications (Fig. 7B). Although the spontaneous contractions were often observed in isolated muscle pieces, they were only rarely observed in innervated I3a preparations. It was necessary to use lower concentrations $(0.1$ $\mu \mathrm{M})$ of the SCPs or 5HT that did not routinely elicit spontaneous contractions to clearly reveal the potentiation of contractions (Fig. 7A). Glutamate-evoked contractions were increased $242 \pm$ $40 \%(n=6)$ over control by the SCPs and $376 \pm 99 \%(n=4)$ by 5HT. The differences between the effects of the SCPs and 5HT were not significant. Although we did not systematically study the time courses of these effects, it appeared that the effects of the SCPs reversed during washout, whereas those of 5HT were persistent.

\section{Effects of the SCPs or 5HT on the latency of contractions}

At a given stimulation frequency, we observed that latency between the onset of the burst in the motor neuron and the evoked contractions was reduced dramatically for B38 and to a much lesser extent for B3 by the SCPs and 5HT (Figs. 8, 11). As for the other effects of these substances, the reduction in latency caused by the SCPs reversed during washout, whereas the reduction caused by 5 HT was persistent (Fig. 8). We performed a series of experiments to determine how the reduction in latencies caused by the SCPs and $5 \mathrm{HT}$ compared with the reduction in latencies caused by increasing the stimulation rate within a burst. Figure 9 shows the results from one such experiment comparing the latencies of B38-evoked contractions at a range of frequencies in ASW and in 5HT. 5HT markedly reduces the threshold frequency required to elicit a contraction (see below) and reduces the latency to an extent similar to that produced by a $\sim 4 \mathrm{~Hz}$ increase in stimulation frequency (compare $12 \mathrm{~Hz}$ in ASW with $8 \mathrm{~Hz}$ in 5HT). The results from similar experiments (Fig. 10) show the effects of the SCPs and 5HT on contractions evoked by B3 and B38. It was not possible to present pooled results, because the threshold frequencies in ASW varied widely between preparations. The SCPs had very little effect on the relationship between frequency and latency for B3 and shifted this relationship $\sim 1 \mathrm{~Hz}$ lower for B38. 5HT shifted this relationship 1-2 Hz lower for B3 and and $\sim 4 \mathrm{~Hz}$ lower for B38. Neither the SCPs nor 5HT appeared to change the slopes of the relationship between frequency and latency, suggesting that they do not modulate frequency-dependent facilitation within a burst.

\section{Effects of the SCPs or 5HT on firing frequencies and burst durations that are threshold for eliciting contractions}

In ASW using $4 \mathrm{sec}$ bursts and $100 \mathrm{sec}$ interburst intervals, the frequencies that were threshold to evoke contractions were $10.0 \pm$ $0.6 \mathrm{~Hz}(n=18)$ for B3 and $11.5 \pm 0.6 \mathrm{~Hz}(n=16)$ for B38. For B38, the threshold frequencies were reduced $\sim 1 \mathrm{~Hz}$ by the SCPs and $\sim 2 \mathrm{~Hz}$ by $5 \mathrm{HT}$ (Fig. 11 for the pooled percentage reduction in frequencies). For B3 the threshold frequencies were essentially unchanged by the SCPs and reduced $\sim 1 \mathrm{~Hz}$ by $5 \mathrm{HT}$.

In the previous experiments, we used $100 \mathrm{sec}$ interburst intervals to minimize the contribution of post-tetanic potentiation to the apparent threshold (Weiss et al., 1978; Lotshaw and Lloyd, 1990). In a second set of experiments, we investigated the effect of reducing this interval from $100 \mathrm{sec}$ to $10 \mathrm{sec}$. Intervals of $10 \mathrm{sec}$ approach the fastest observed feeding cycles in behavioral exper-

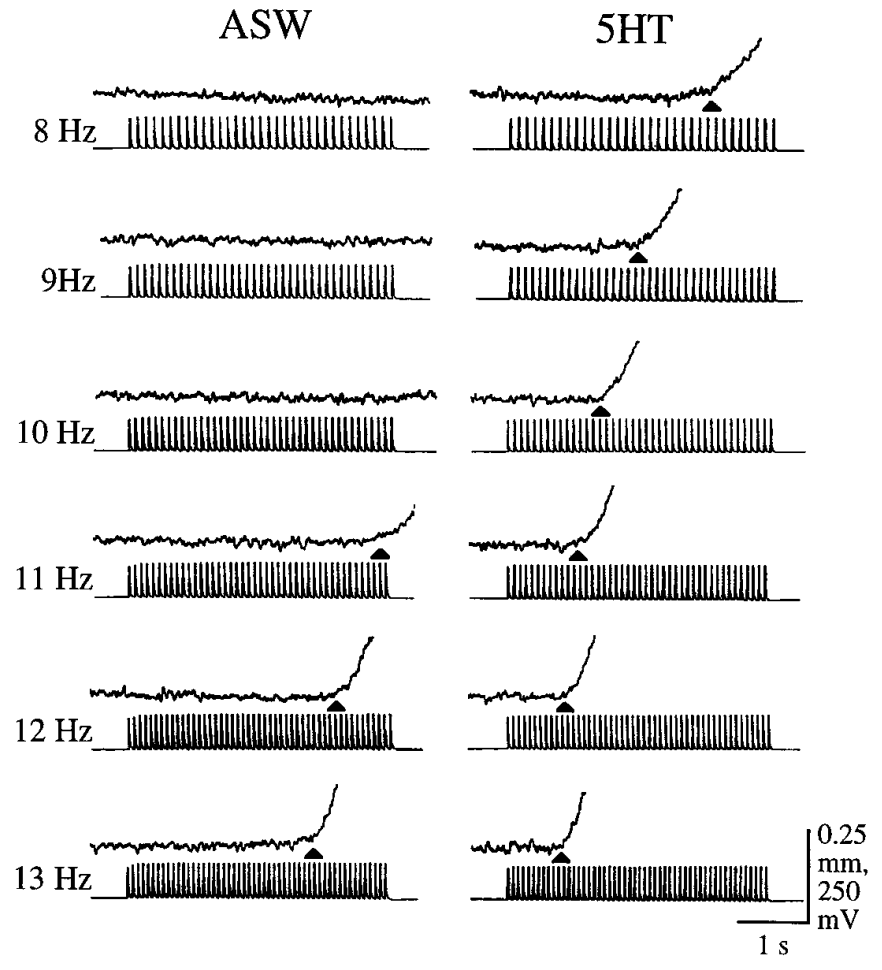

Figure 9. Effects of firing frequency and 5HT on the latency between the onset of a B38-evoked burst and the onset of the resulting I3a contraction. Contractions were evoked by alternately stimulating B3 or B38 (4 sec bursts at interburst interval of $100 \mathrm{sec}$ ). Only recordings from B38 are shown. Stimulation frequency was increased 1 spike/sec $(\mathrm{Hz})$ during the interburst intervals and is indicated next to the motor neuron burst. Superfusion with $1 \mu \mathrm{M}$ 5HT dramatically decreased the latency between the onset of the B38-evoked burst and the onset of the contraction. Note that 5 HT reduced the latency to an extent similar to that produced by $\sim 4$ $\mathrm{Hz}$ increase in stimulation frequency (compare $12 \mathrm{~Hz}$ in ASW with $8 \mathrm{~Hz}$ in 5HT). The first contraction at each frequency is in ASW, and the second is after 10 min superfusion with $5 \mathrm{HT}$, so the effects are nearly maximal (see Fig. 5). Black arrowheads indicate the onset of the contraction.

iments and thus probably represent the shortest natural interburst interval for B38 (Kupfermann, 1974; Cropper et al., 1990). After determining the thresholds for $4 \mathrm{sec}$ bursts at $100 \mathrm{sec}$ intervals, the thresholds were determined for a series of $4 \mathrm{sec}$ bursts at 10 sec intervals. The reduction in interburst interval had no effect on threshold frequency for B3 (reduced by $1 \pm 7 \% ; n=5$ ) and only moderately reduced this frequency for B38 (reduced by $9 \pm 3 \%$; $n=5$ ). For B38, this reduction in threshold frequency corresponds to $\sim 1 \mathrm{~Hz}$. It should be emphasized that the mechanisms underlying this post-tetanic potentiation may well include release of the SCPs from B38 terminals. Indeed, there is evidence that stimulation of $\mathrm{B} 38$ at $10 \mathrm{~Hz}$ releases the SCPs, and it is possible that release occurs at even lower frequencies (Church et al., 1993).

We also investigated the effects of the SCPs and 5HT on burst durations necessary to evoke contractions. When stimulated at 16 $\mathrm{Hz}$ in ASW, the mean burst durations that were just above threshold for contractions were $1.3 \pm 0.1 \mathrm{sec}(n=5)$ for B3 and $2.1 \pm 0.2 \mathrm{sec}(n=5)$ for B38. For B38, threshold burst durations were decreased to a similar degree by the SCPs and 5HT (Fig. 11). This corresponded to an average decrease of $1.0 \pm 0.2 \mathrm{sec}$ $(n=5)$ for the SCPs and $1.4 \pm 0.1 \mathrm{sec}$ for 5HT. For B3, threshold burst durations were not reduced by the SCPs and were only 

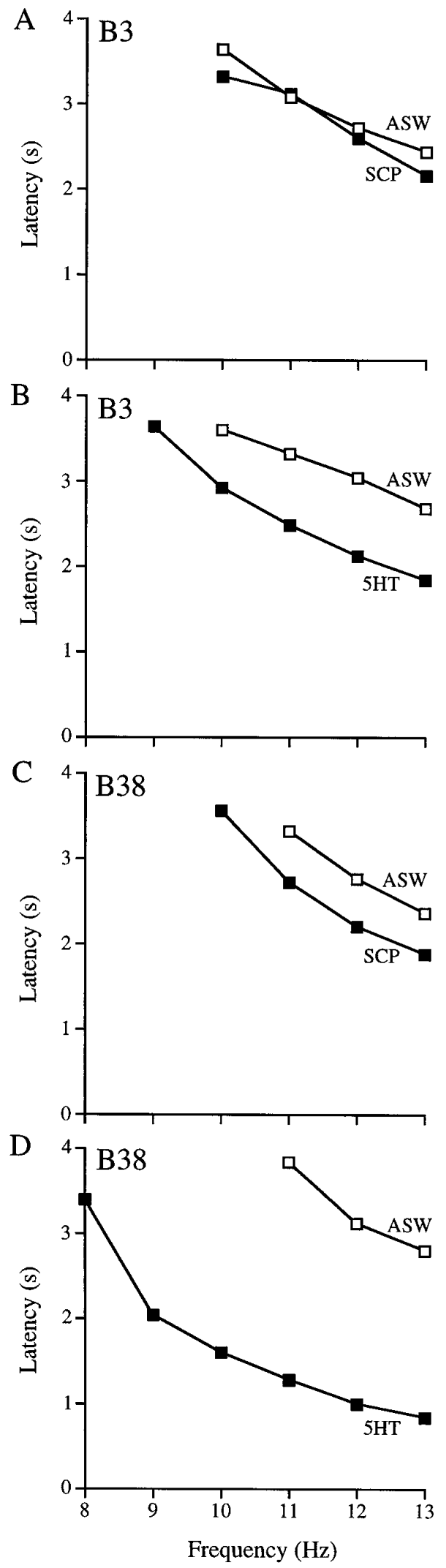

Figure 10. Effects of the SCPs and 5HT on the latency of contractions evoked by firing the motor neuron at different frequencies. Contractions were evoked by alternately stimulating B3 or B38 (4 sec bursts at interburst interval of $100 \mathrm{sec}$ ) at frequencies between 8 and $13 \mathrm{~Hz}$. Stimulation frequency was increased $1 \mathrm{spike} / \mathrm{sec}(\mathrm{Hz})$ during the interburst intervals slightly reduced by $5 \mathrm{HT}(0.1 \pm 0.1 \mathrm{sec}, n=5)$. Thus, the SCPs and 5HT had little effect on the threshold burst durations for B3-evoked contractions but markedly reduced burst durations required for B38-evoked contractions (Fig. 11). As was observed for other modulatory effects, the actions of the SCPs on threshold frequencies and threshold burst durations reversed during washout, whereas those of 5HT were persistent (data not shown).

Because the SCPs and 5HT had very small and similar effects on B3-evoked EJPs (Fig. 4), it appears that the effects of 5HT on the properties of B3-evoked contractions were largely through its effects on excitation-contraction coupling. For B3-evoked contractions, 5HT was more effective at decreasing threshold firing frequency and contraction latency than at reducing threshold burst duration. This suggests that increased efficiency of excitation-contraction coupling has a significant effect on threshold firing frequency and little effect on threshold burst duration.

\section{DISCUSSION}

In the present study, we investigated the actions of both intrinsic and extrinsic modulatory transmitters in a preparation consisting of muscle fibers that are innervated by two excitatory motor neurons, which likely use glutamate as their fast excitatory transmitter, and a modulatory serotonergic neuron. Individual fibers are functionally innervated by both B3 and B38. The SCPs and 5 HT selectively facilitated B38-evoked EJPs. This selective facilitation could have a presynaptic site of action, increasing the release of glutamate, or a postsynaptic site of action, increasing the receptor sensitivity specifically at receptors that bind glutamate released from $\mathrm{B} 38$ but not $\mathrm{B} 3$, or could involve changes at both sites. In any case, because the SCPs are normally released from terminals of B38, they would reach their highest concentrations in precisely the region necessary to selectively facilitate synaptic potentials of B38 (Lotshaw and Lloyd, 1990; Church et al., 1993).

We next tested how the facilitation of EJPs effected muscle contractions. Both the SCPs and 5HT potentiated contractions, and there was a trend for the SCPs and 5HT to potentiate contractions evoked by B38 more than those evoked by B3, but the differences were much smaller than expected from the differences in facilitation of the EJPs. An explanation for this is that the SCPs and 5HT also modulate some step(s) in excitationcontraction coupling so that even the largely unchanged B3evoked EJPs produce larger contractions. Another finding that supports the suggestion that there are postsynaptic effects of the SCPs and 5HT in the muscle fibers is the observation that they elevate cAMP levels in I3a neuromuscular preparation up to 20 -fold over control values (Lotshaw and Lloyd, 1990). These increased levels must occur primarily in muscle fibers, because they represent nearly the entire tissue volume of the muscle. In the present study, three additional observations indicate that the

$\leftarrow$

preceding the B38-evoked bursts. It was not possible to present pooled results because the threshold frequencies varied widely between preparations, so representative examples are shown. $A, \operatorname{SCP}_{\mathrm{A}}(1 \mu \mathrm{M})$ had little effect on the relationship between frequency and latency for B3evoked contractions. $B$, 5HT $(1 \mu \mathrm{M})$ shifted this relationship 1-2 Hz lower for $\mathrm{B} 3 . C, \mathrm{SCP}_{\mathrm{A}}(1 \mu \mathrm{M})$ shifted this relationship between frequency and latency $\sim 1 \mathrm{~Hz}$ for B38-evoked contractions. $D$, 5HT $(1 \mu \mathrm{M})$ shifted this relationship $\sim 4 \mathrm{~Hz}$ lower for B38. Note that the SCPs and $5 \mathrm{HT}$ did not change the slope of the relationship between frequency and latency, suggesting that they do not modulate frequency-dependent facilitation within a burst. 

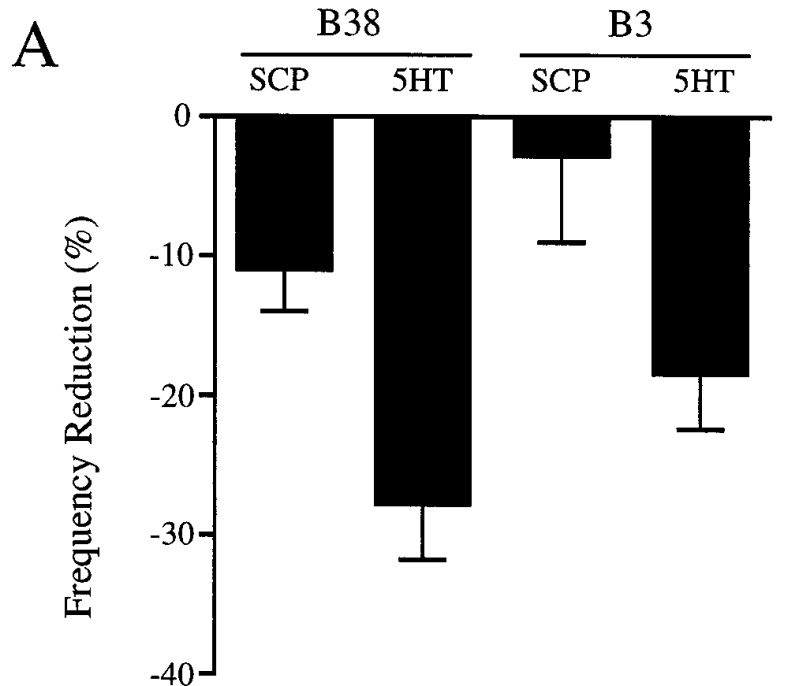

$\mathrm{B}$
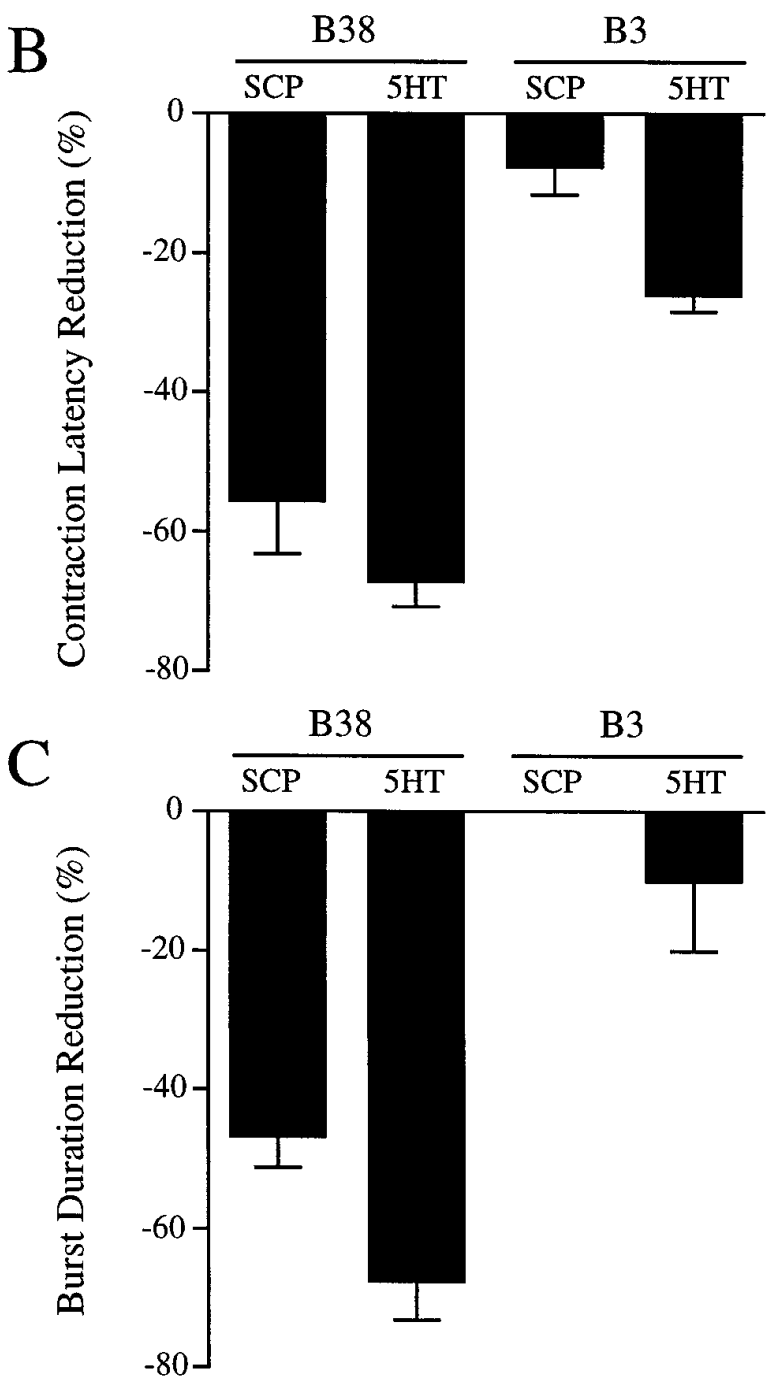

Figure 11. Summary of the effects of $1 \mu \mathrm{M}$ SCPs and $1 \mu \mathrm{M}$ 5HT on (1) the threshold firing frequency required to elicit contractions, (2) the latency between the onset of the burst and onset of the evoked contraction, and (3) the threshold burst duration required to elicit contractions. $A$, The threshold firing frequency to elicit $\mathrm{B} 38$-evoked contractions was reduced by the SCPs and 5HT $(n=3 ; p \leq 0.01 ; t$ test). The threshold firing frequency for $\mathrm{B} 3$ was reduced by $5 \mathrm{HT}(n=3 ; p \leq 0.01)$, whereas the effect of the SCPs was not significant $(n=3)$. $B$, The latency between
SCPs and 5HT have postsynaptic effects: (1) they potentiated contractions evoked by the application of fixed amounts of exogenous glutamate as well as increased overall excitability of the muscle fibers; (2) they increased contraction relaxation rates; and (3) they decreased EJP decay time constants, which reflect a decrease in the input resistance of muscle fibers.

Our interpretation of these results is that the potentiation of contractions produced by the SCPs and 5HT is mediated through at least two processes: the facilitation of B38-evoked EJPs, and additional processes in excitation-contraction coupling that enhance contractions evoked by both B3 and B38. There were also differences between the effects of the SCPs and 5HT on contractions. 5HT was more effective at potentiating the contractions than the SCPs (see below). Many of the effects of the SCPs and 5 HT on buccal muscles are thought to be mediated via increased cAMP levels (Weiss et al., 1979; Lloyd et al., 1984; Brezina et al., 1994; Hooper et al., 1994; Probst et al., 1994). At $1 \mu \mathrm{M}$, however, the SCPs and 5HT produce very similar increases of cAMP in I3a muscles (Lotshaw and Lloyd, 1990). Thus, it is possible that the greater effectiveness of 5HT on excitation-contraction coupling may involve another second messenger system, as described for the serotonergic facilitation of central synapses in Aplysia (Byrne and Kandel, 1996). It is also possible that activation of another second messenger system may underlie the persistence of the effects of 5HT on the I3a muscle.

It is interesting to compare our results with those obtained for another well studied buccal muscle (termed the ARC or I5). The SCPs or 5HT increase the amplitude and relaxation rates of contractions in this muscle but have only small effects on the amplitude of EJPs evoked by either of the motor neurons that innervate the muscle. Levels of cAMP in the muscle fibers are elevated substantially and to a similar extent by the SCPs and 5HT (at $1 \mu \mathrm{M}$ ) (Lloyd et al., 1984; Brezina at al., 1994). Evidence from voltage-clamp analyses of isolated fibers from this muscle indicates that the SCPs and 5HT largely mediate their effects on contraction amplitude by enhancing a voltage-dependent calcium current that apparently provides all or most of the calcium required for contractions (Brezina and Weiss, 1993; Brezina at al., 1994). An interesting result from these studies was that at $1 \mu \mathrm{M}$, 5 HT caused twice the increase in calcium current as that caused by the SCPs. If a similar current was present in I3a muscle fibers, this could explain the differences in the potentiation of contractions observed between the SCPs and 5HT.

The effects of 5HT on B38-evoked EJPs and contractions are also similar to those observed previously in crustacean preparations. At both crayfish and lobster skeletal muscles, 5HT has been shown to facilitate EJPs (Dudel, 1965; Kravitz et al., 1980; Glusman and Kravitz, 1982; Dixon and Atwood, 1985). This effect has been shown to be mediated by an increase in transmitter release, perhaps involving a change in presynaptic calcium metabolism. Furthermore, the effects of 5HT reversed during washout in two phases: one rapid and one much slower, although still much less

$\leftarrow$

the onset of the burst and onset of the B38-evoked contraction was reduced by the SCPs and 5HT $(n=5 ; p \leq 0.01)$. This latency for B3 was also reduced by 5 HT $(n=6 ; p \leq 0.01)$, whereas the effect of the SCPs was not significant $(n=6)$. $C$, The threshold burst duration to elicit contractions was reduced by the SCPs and 5HT for B38-evoked contractions $(n=5 ; p \leq 0.01)$. Note that the SCPs and 5HT were more effective at reducing contraction latency and threshold burst duration for B38-evoked contractions than for B3-evoked contractions $(p \leq 0.01$; paired $t$ test) 
persistent than we observed in the I3a muscle. In addition to the facilitated EJPs, 5HT also has an effect on muscle contractility in lobster similar to what we observed (Harris-Warrick and Kravitz, 1984). Finally, the actions of 5HT on crustacean neuromuscular synapses appear to be mediated through increased cAMP levels as well as by other second messenger systems (Dixon and Atwood, 1989a,b).

Contractions are triggered in buccal muscle fibers when EJPs depolarize muscle fibers sufficiently to activate a voltage-gated calcium current (Brezina et al., 1994). At a given firing frequency, the facilitation of B38-evoked EJPs might shorten the time required to depolarize fibers into the voltage region where calcium current is activated and thereby decrease the latency between the onset of a burst and the onset of a contraction, it might permit shorter duration bursts to elicit contractions, and it might reduce the firing frequency required to depolarize fibers into the voltage region at a given burst duration. Indeed, both the SCPs and 5HT reduced all three parameters much more for contractions evoked by B38 than by B3.

In summary, there are several important behavioral consequences of the modulation of I3a muscle contractions by the SCPs and 5HT. These include (1) large increases in the amplitude and the rate of relaxation of contractions, (2) a decrease in the firing frequency required to elicit a contraction, (3) a decrease in the burst duration required to elicit a contraction, and (4) a decrease in the latency between the onset of a burst and the onset of the contraction. The effects on contraction amplitude and relaxation rates were roughly similar for contractions evoked by B3 and B38; however, the effects on threshold burst duration and contraction latency were more pronounced for B38, presumably reflecting the contribution of the facilitated EJPs. These effects would be expected to have several consequences for B38-evoked contractions, which would be important during feeding behavior. During ingestive-like motor programs evoked by stimulation of an interneuron, B3 fires at a frequency of up to $20 \mathrm{~Hz}$ in $\sim 6 \mathrm{sec}$ bursts, whereas B38 fires at a frequency of up to $10 \mathrm{~Hz}$ in $\sim 4 \mathrm{sec}$ bursts (Church and Lloyd, 1994). Using $4 \mathrm{sec}$ bursts, we found that the threshold firing frequency to elicit contractions was $\sim 10 \mathrm{~Hz}$ for B3 and $\sim 12 \mathrm{~Hz}$ for B38. Thus, using interburst intervals of 100 sec, the B3 bursts would be well above threshold for contractions, but those of B38 would be at or below threshold. Release of the SCPs or 5HT would increase the likelihood that bursts in B38 would be above threshold for contractions. This is particularly important functionally for B38, because no other identified excitatory motor neurons that innervate the same fibers were found to fire at the same time as B38. Indeed, bursts in B38 partially overlap with the inhibitory motor neuron B47, which would further reduce the effectiveness of $\mathrm{B} 38$ at eliciting contractions (Church and Lloyd, 1994). By contrast, a number of excitatory motor neurons fire with B3 and have similar fields of innervation. During feeding, the cycle period and presumably the interburst interval for B38 is often much shorter than $100 \mathrm{sec}$, and we did observe a reduction in threshold frequency when interburst intervals were reduced to $10 \mathrm{sec}$; however, some of this effect might be attributable to release of endogenous SCPs. The reduction in contraction latency may be important during feeding behavior. Behavioral experiments indicate that the period of ingestive motor programs may be as short as $8 \mathrm{sec}$, so that changes in the timing on the order of a few seconds as were observed for the effects of the SCPs and 5HT on B38-evoked contraction latencies would have major implications for behavioral output. The increased rate of relaxation of contractions evoked by both B3 and
B38 may also be important in permitting rapid cycling of buccal muscle contractions (Weiss et al., 1992).

\section{REFERENCES}

Brezina V, Weiss KR (1993) Analysis of ion currents mediating modulation of the ARC muscle of Aplysia by simultaneous on-line length measurements and current/voltage clamp. Soc Neurosci Abstr 19:1262.

Brezina V, Evans CG, Weiss KR (1994) Enhancement of Ca current in the accessory radula closer muscle of Aplysia californica by neuromodulators that potentiate contractions. J Neurosci 14:4393-4411.

Brezina V, Orekhova IV, Weiss KR (1996) Functional uncoupling of linked transmitter effects by combinatorial convergence. Science 273:806-810.

Byrne JH, Kandel ER (1996) Presynaptic facilitation revisited: state and time dependence. J Neurosci 16:425-435.

Calabrese RL (1989) Modulation of muscle and neuromuscular junctions in invertebrates. Semin Neurosci 1:25-34.

Church PJ, Lloyd PE (1991) Expression of diverse neuropeptide cotransmitters by identified motor neurons in Aplysia. J Neurosci 11:618-625.

Church PJ, Lloyd PE (1994) Activity of multiple identified motor neurons recorded intracellularly during evoked feeding-like motor programs in Aplysia. J Neurophysiol 72:1794-1809.

Church PJ, Whim MD, Lloyd PE (1993) Modulation of neuromuscular transmission by conventional and transmitters released from excitatory and inhibitory motor neurons in Aplysia. J Neurosci 13:2790-2800.

Cohen JL, Weiss KR, Kupfermann I (1978) Motor control of buccal muscles in Aplysia. J Neurophysiol 41:157-180.

Cropper EC, Lloyd PE, Reed W, Tenenbaum R, Kupfermann I, Weiss KR (1987a) Multiple neuropeptides in cholinergic motor neurons of Aplysia: evidence for modulation intrinsic to the motor circuit. Proc Natl Acad Sci USA 84:3486-3490.

Cropper EC, Tenenbaum R, Gawinowicz Kolks MA, Kupfermann I, Weiss KR (1987b) Myomodulin: a bioactive neuropeptide present in an identified cholinergic buccal motor neuron of Aplysia. Proc Natl Acad Sci USA 84:5483-5486.

Cropper EC, Miller MW, Tenenbaum R, Gawinowicz Kolks MA, Kupfermann I, Weiss KR (1988) Structure and action of buccalin A: a modulatory neuropeptide localized to an identified small cardioactive peptide-containing cholinergic motor neuron of Aplysia californica. Proc Natl Acad Sci USA 85:6177-6181.

Cropper EC, Price D, Tenenbaum R, Kupfermann I, Weiss KR (1990) Release of peptide cotransmitters from a cholinergic neuron under physiological conditions. Proc Natl Acad Sci USA 87:933-937.

Cropper EC, Brezina V, Vilim FS, Harish O, Price DA, Rosen S, Kupfermann I, Weiss KR (1994) FRF peptides in the ARC neuromuscular system of Aplysia: purification and physiological actions. J Neurophysiol 72:2181-2195.

Dixon D, Atwood HL (1985) Crayfish motor nerve terminal's response to serotonin examined by intracellular electrode. J Neurobiol 16:409-424.

Dixon D, Atwood HL (1989a) Phosphatidylinositol system's role in serotonin-induced facilitation at the crayfish neuromuscular junction. J Neurophysiol 62:239-246.

Dixon D, Atwood HL (1989b) Conjoint action of phosphatidylinositol and adenylate cyclase systems in serotonin-induced facilitation at the crayfish neuromuscular junction. J Neurophysiol 62:1251-1259.

Dudel J (1965) Facilitatory effects of 5-hydroxytryptamine on the crayfish neuromuscular junction. Naunyn Schmiedebergs Arch Pathol Pharmacol 249:515-528.

Fox LE, Lloyd PE (1993) Evidence that L-glutamate is the excitatory transmitter to buccal muscle I3 in Aplysia. Soc Neurosci Abstr 19:490.

Gardner D (1971) Bilateral symmetry and interneuronal organization in the buccal ganglia of Aplysia. Science 173:550-553.

Glusman S, Kravitz EA (1982) The action of serotonin on excitatory nerve terminals in lobster nerve-muscle preparations. J Physiol (Lond) 325:223-241.

Harris-Warrick RM, Kravitz EA (1984) Cellular mechanisms for modulation of posture by octopamine and serotonin in lobster. J Neurosci 4:1976-1993.

Hooper SL, Probst WC, Cropper EC, Kupfermann I, Weiss KR (1994) SCP application or B15 stimulation activates CAPK in the ARC muscle of Aplysia. Brain Res 657:337-341.

Howells HH (1942) The structure and function of the alimentary canal of Aplysia punctata. Q J Microscop Sci 83:357-397. 
Kravitz EA, Glusman S, Harris-Warrick RM, Livingstone MS, Schwartz T, Goy MF (1980) Amines and a peptide as neurohormones in lobsters: actions on neuromuscular preparations and preliminary behavioural studies. J Exp Biol 89:159-176.

Kupfermann I (1974) Feeding behavior in Aplysia: a simple system for the study of motivation. Behav Biol 10:1-26.

Lloyd PE (1986) The small cardioactive peptides: a class of modulatory neuropeptides in Aplysia. Trends Neurosci 9:428-431.

Lloyd PE (1988) Fast axonal transport of modulatory neuropeptides from central ganglia to components of the feeding system in Aplysia. J Neurosci 8:3507-3514.

Lloyd PE, Church PJ (1994) Cholinergic neuromuscular synapses in Aplysia have low endogenous acetylcholinesterase and a high affinity uptake system for acetylcholine. J Neurosci 14:6722-6733.

Lloyd PE, Kupfermann I, Weiss KR (1984) Evidence for parallel actions of a molluscan peptide $\left(\mathrm{SCP}_{\mathrm{B}}\right)$ and $5 \mathrm{HT}$ in mediating arousal in Aplysia. Proc Natl Acad Sci USA 81:2934-2937.

Lloyd PE, Frankfurt M, Stevens P, Kupfermann I, Weiss KR (1987) Biochemical and immunocytological localization of the neuropeptides FMRFamide, $\mathrm{SCP}_{\mathrm{A}}$, and $\mathrm{SCP}_{\mathrm{B}}$ to neurons involved in the regulation of feeding in Aplysia. J Neurosci 7:1123-1132.

Lotshaw DP, Lloyd PE (1990) Peptidergic and serotonergic facilitation of a neuromuscular synapse in Aplysia. Brain Res 526:81-94.
Probst WC, Cropper EC, Heierhorst J, Hooper SL, Jaffe H, Vilim F, Beushausen S, Kupfermann I, Weiss KR (1994) cAMP-dependent phosphorylation of Aplysia twitchin may mediate modulation of muscle contractions by neuropeptide cotransmitters. Proc Natl Acad Sci USA 91:8487-8491.

Weiss KR, Cohen JL, Kupfermann I (1978) Modulatory control of buccal musculature by a serotonergic neuron (metacerebral cell) in Aplysia. J Neurophysiol 41:181-203.

Weiss KR, Mandelbaum DE, Schonberg M, Kupfermann I (1979) Modulation of buccal muscle contractility by serotonergic metacerebral cells in Aplysia: evidence for a role of cyclic adenosine monophosphate. J Neurophysiol 42:791-803.

Weiss KR, Brezina V, Cropper EC, Hooper SL, Miller MW, Probst WC, Vilim FS, Kupfermann I (1992) Peptidergic co-transmission in Aplysia: functional implications for rhythmic behaviors. Experientia 48:456-463.

Whim MD, Lloyd PE (1989) Frequency-dependent release of peptide cotransmitters from identified cholinergic motor neurons in Aplysia. Proc Natl Acad Sci USA 86:9034-9038.

Whim MD, Lloyd PE (1990) Neuropeptide cotransmitters released from an identified cholinergic motor neuron modulate neuromuscular efficacy in Aplysia. J Neurosci 10:3313-3322. 